\title{
A NOVEL MOBILITY AID FOR THE BLIND THROUGH ULTRASOUND PULSED ECHO MODULATED NERVE STIMULATION
}

\author{
M Shamiul Fahad ${ }^{1}$, M Muwyid Uzzaman Khan ${ }^{1}$ and K Siddique-e-Rabbani
}

\author{
Department of Biomedical Physics \& Technology, University of Dhaka, Dhaka, Bangladesh \\ email: f.nibir@gmail.com,mueid_khan@hotmail.com, rabbani@univdhaka.edu
}

\begin{abstract}
There are no affordable and widely available technology based aid to give a blind person the power of free movement avoiding obstacles and dangers. Some ultrasound pulse echo based electronic aids deliver the information obtained through the echo through audio signals conveyed by means of ear phones, but these affect the capability of such an important sense organ which a blind person uses greatly to receive and discern information of the surroundings. In the present work this limitation is overcome by passing this information through nerve stimulations at a suitable point on the body, possible wrist. Here the delays of the received ultrasound echo-pulses are used to modulate the frequency of nerve stimulation, to give higher frequency for shorter distances. This was designed to match the common psychological response of attributing higher frequencies to danger, creating a natural reaction for moving away when an object is too close. The highest frequency a human nerve can be stimulated to is about $500 \mathrm{~Hz}$, and pulses with heights of $100 \mathrm{~V}$ to $200 \mathrm{~V}$ are required for stimulating nerves passing just under the skin using surface electrodes. Necessary electronic circuit design was developed and a prototype fabricated in the present work. The prototype was tested for short distances up to $20 \mathrm{~cm}$ giving very good linear response between object distance and the stimulating frequency, with the inverse characteristics as mentioned above. Reduction of noise through proper housing and shielding will allow the device to be useful to a practical distance of about $10 \mathrm{~m}$ using $40 \mathrm{kHz}$ ultrasound transducers.
\end{abstract}

Keywords: Ultrasound, transducer, echolocation, surface electrodes, nerve stimulation.

\section{INTRODUCTION}

A blind person has to depend mainly on other senses, particularly of hearing ability to get information of the surroundings. Modern technology has given a blind person the ability to read through touch, but not much is available to give him or her freedom in movement except the common white stick. Of course, this stick is more of a flag to inform others rather than for the freedom of movement to the blind person him or herself. A stick allows information of the presence of obstacles over a very small range around the person and the process is also very slow and cumbersome. Unfortunately no affordable commercial device is widely available based on modern technology to help blind people in their movement. Some attempted designs use ultrasound pulse echo techniques, as employed by bats and dolphins, and the information is passed on to the user through sound signals delivered through earphones [1-4]. The ability of hearing is very important to a blind person as the person uses this to compensate for the loss of sight. Therefore, this method has the disadvantage that it impairs the natural hearing ability of the person through the extra stimuli coming from this new device, cluttering the normal sound. Besides, the presence of the earphone itself poses obstruction to the free flow of sound to the ear. Therefore engaging the precious hearing ability to receive information through such an artificial device will rob the person of some important information that s/he could have otherwise obtained and deciphered. Therefore we conceived of giving the information through means other than hearing.

\footnotetext{
${ }^{1}$ Department of Electrical \& Electronic Engineering, Islamic University of Technology Gazipur, Bangladesh
} 
On a different application setting, nerve stimulation at wrist as an aid for the profoundly deaf was attempted by a group at Sheffield [5]. This means of delivery appeared to us as a sensible choice in the present application which will keep the faculty of hearing undisturbed.

Thus the present idea was based on a mixture of the above two, originally attempted for two different applications. In the present work ultrasound pulse echo would be used to get information of the surroundings while the information would be presented to the subject through nerve stimulations at a suitable location [6]. Moreover, further information would be provided on the distance of an obstacle through variation of frequency of stimulation. This will allow the user to identify stationary or moving objects as well, and to assess whether the object is approaching or moving away, and to take necessary safety measures when something comes dangerously close. In a practical implementation of the idea, the sensors would be mounted on the sides of a sun glass or a spectacle so that by turning the head around, the user can also get information on the direction of the object.

\section{METHODS}

\subsection{Basic design concepts}

The basic idea is described with the help of Fig.1. An ultrasound pulse generated in the device will be transmitted through air and a pulse (echo) will return through reflection from an obstacle in the path. The time delay $t_{d}$ between the transmitted and the received pulses, being twice the time taken for one way travel, will give the distance of the object from the velocity of sound in air at the particular temperature. That is, $t_{d}$ will be larger for greater distance of object. On the receiver side, a nerve will be stimulated at a frequency $f$ based on the echo time delay $t_{d}$. As mentioned before, an increasing stimulation frequency is desired for decreasing distance to the object. This will give a normal psychological reaction to move away from the path if the frequency is too high, representing an object too close. The desired relationship between $f$ and $t_{d}$ is depicted in Fig. 1 and is given by,

$$
f=f_{\max }-k \times t_{d}
$$

Where $f_{\max }$ is the chosen maximum frequency of stimulation and $-k$ is the slope of the straight line. When the time delay is extremely short it should imply that the blind person is about to hit an obstacle right in front. However, the highest frequency that can be applied to a human nerve for creating a sensation is about $500 \mathrm{~Hz}$ and this determines $f_{\max }$ in this application.

For nerve stimulation high voltage pulses (height about 100V, width about $1 \mathrm{msec}$ ) will be produced to stimulate a nerve running close to the skin using surface electrodes, and the frequency $f$ of this stimulation will be modified according to the above requirement.

For safe movement a blind person should be able to sense the presence of objects at distances of about 5 to $10 \mathrm{~m}$. For this requirement an ultrasound frequency of $40 \mathrm{kHz}$ was chosen because at higher frequencies, ultrasound gets attenuated in air quickly and the sensitive range is reduced. In the scheme proposed and developed in the present work, the wearer would not only be able to sense the presence of an object in the neighbourhood, but would also be able to sense the direction of movement of object, whether it is moving towards
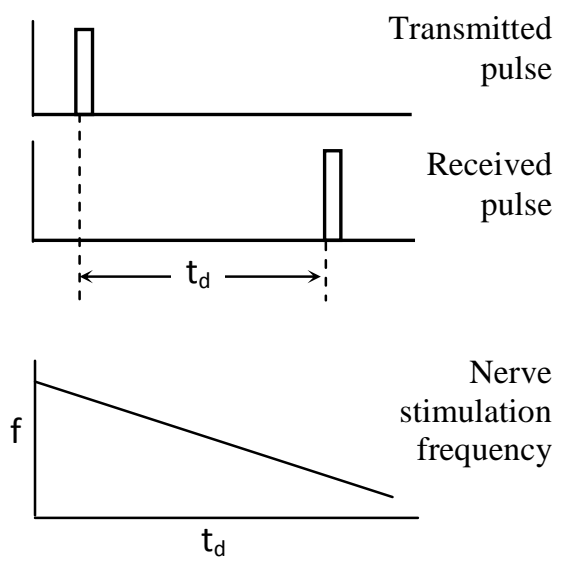

Fig.1: Time delay of ultrasound echo $t_{d}$ to control frequency of nerve stimulation, higher for smaller $t_{d}$ 
or away from the wearer. After one pulse is transmitted the wearer has to wait till an echo is received for the greatest range desired for this device. In the present work, a range of $10 \mathrm{~m}$ was thought to be the maximum desired from a practical point of view. At a velocity of $330 \mathrm{~m} / \mathrm{sec}$ for sound in air, this will require about $66 \mathrm{msec}$ for the echo to be received (a total distance of $20 \mathrm{~m}$ both way). Therefore the interval between subsequent pulses should be more than $66 \mathrm{msec}$.

On a separate requirement, it is desired that the user gets the information frequently so that he or she can take a quick decision. Ideally continuous real time information is desired. However, in practice, information received several times a second would be adequate, and may be termed real time. In the present work, a refresh rate of about 6 times a second was thought to be adequate for the ultrasound pulses corresponding to a pulse repetition interval of about $150 \mathrm{msec}$. This satisfies the previous requirement and was chosen for the present prototype.

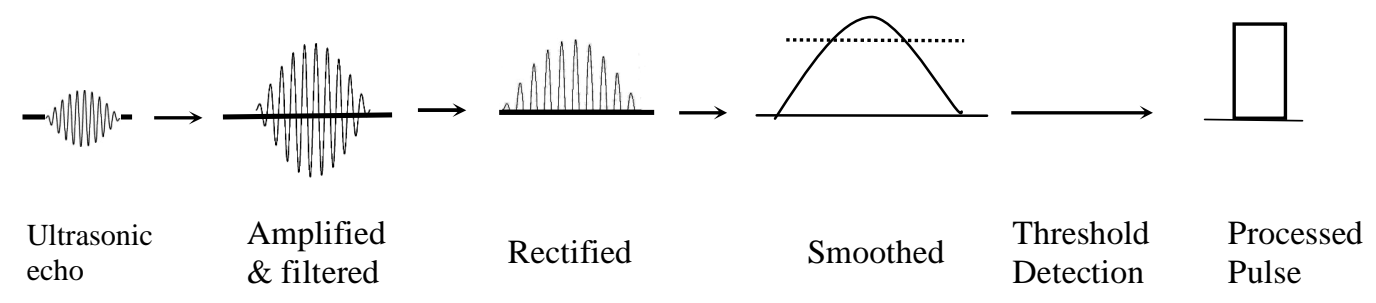

Fig.2: Scheme of deriving an electrical pulse from a received ultrasound burst pulse.

\subsection{Generating and Receiving Ultrasound Pulses}

Commercially available $40 \mathrm{kHz}$ piezoelectric transducers were used for this purpose, one acting as the transmitter and the other, the receiver. Both of these devices have resonances at the same value of $40 \mathrm{kHz}$. To generate ultrasound pulses, a sharp spike like rectangular electrical pulse is applied to the transducer which produces damped sinusoidal ultrasound waves at $40 \mathrm{kHz}$ with a sharp rise and a slow decay. On receiving this ultrasound pulsed wave, the receiving transducer produces a short burst of electrical sinusoidal signal at $40 \mathrm{kHz}$. However, because of mechanical inertia, the received pulses gradually build up in amplitude, and then decay gradually since the incident wave itself is decaying slowly, producing a burst of waves as shown in Fig.2. To reproduce a rectangular pulse this wave burst is rectified and smoothed to produce a rounded dc pulse. Finally using a threshold detector in the form of a Schmitt trigger a rectangular pulse is obtained. This whole scheme is depicted in Fig.2.

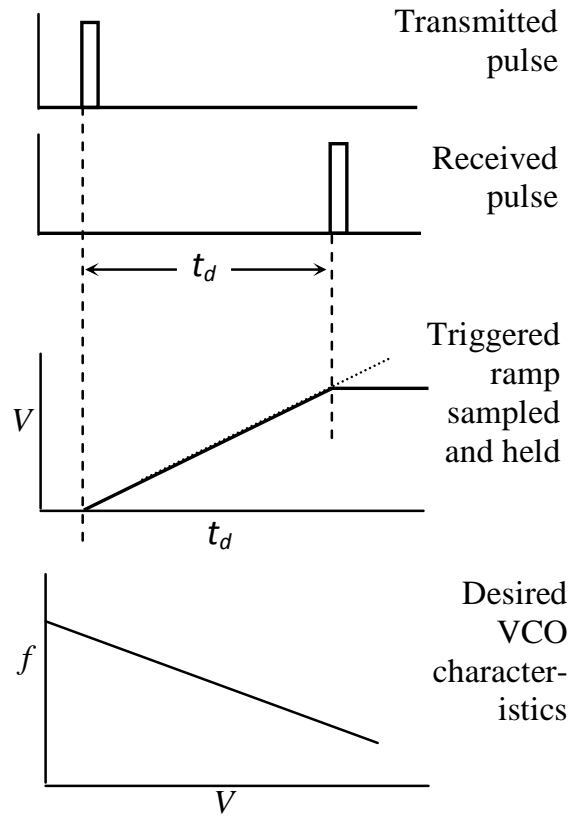

Fig.3: Obtaining a dc voltage to correspond to $t_{d}$ and the VCO frequency characteristics for nerve stimulation 


\subsection{Extracting Time Delay $\mathbf{T}_{d}$ Electronically}

Extracting the time delay $t_{d}$ from the transmitted and received pulse electronically needs some specialised scheme which is depicted in Fig.3. A triggered ramp generator in initiated by a trigger from the US transmitter pulse generator circuitry. The voltage of this ramp at any point of time is proportional to the time delay since the occurrence of the transmitter pulse. When a received pulse arrives, the ramp voltage is sampled and held at that voltage using a 'sample and hold' circuit. Then this voltage will be proportional to the time delay $t_{d}$.

\subsection{Generating Control Pulses For Nerve Stimulation}

As mentioned before, a greater frequency of stimulation is desired for a shorter $t_{d}$, i.e., for a smaller $V$ at the output of the 'sample and hold' circuit mentioned above. That means a voltage controlled oscillator (VCO) circuitry is required whose frequency decreases linearly with increasing control voltage as shown in the lowest part of Fig.3.

\subsection{The Total Hardware Scheme}

The basic design of the whole system is presented with the help of Fig.4. The Pulse generator is an astable or free running rectangular pulse oscillator having a very small pulse width (less than the time period of the $40 \mathrm{kHz}$ ultrasound signal used), and a repetition frequency of about $6 \mathrm{~Hz}$. This drives an ultrasound (US) transmitting transducer. The transmitted ultrasound travels through air and returns an echo pulse if an obstacle falls on the way. The US receiving transducer produces an electrical signal which is amplified, filtered, rectified and threshold detected to produce a rectangular pulse as depicted in Fig.2 before.

The pulse generator also triggers a voltage ramp generator whose output voltage increases linearly with time from the instant of the trigger as depicted in Fig.3. This is taken to one of the inputs of a 'sample and hold' circuit whose other input is the trigger from the received and processed US pulse. When a US echo pulse is received, the 'sample and hold' circuit samples the value of the ramp voltage at that instant and holds that value constant for some time.

A voltage controlled oscillator (VCO) was designed to give a low frequency for a high voltage and vice versa as depicted in Fig.3. This VCO gets an input from the output voltage of the 'sample and hold' circuit. However, the voltage had to be channeled

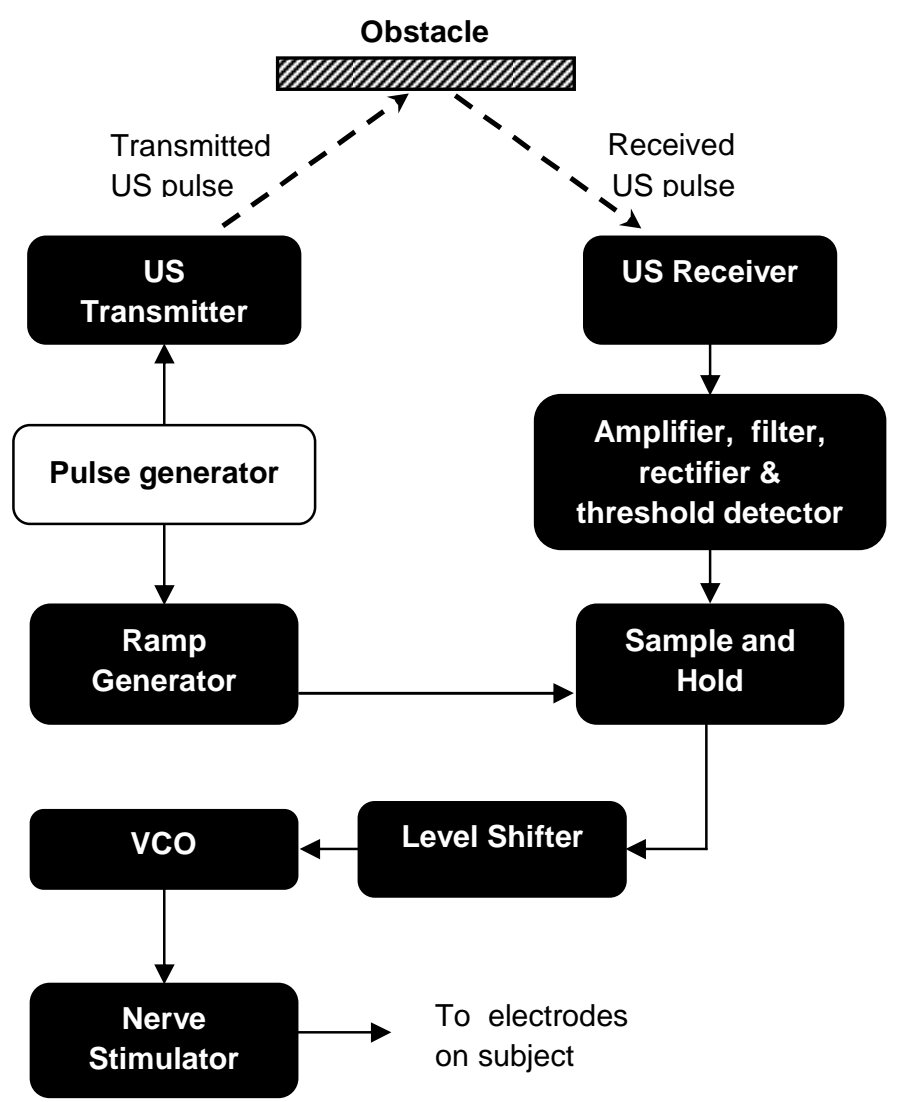

Fig.4: Block diagram of the whole blind mobility aid system 
through a level shifter circuit to suit the VCO parameters. The VCO gave square wave forms which were used to activate a nerve stimulator circuit. This produced the necessary high voltage pulses to stimulate a nerve using surface electrodes. Thus a longer time delay of echo gives rise to a higher voltage at the 'sample and hold' output resulting in a lower frequency of nerve stimulation and vice versa.

\subsection{Actual Circuit Implementation}

The developed circuit is shown in Fig.5 and a brief description is given below. It needs to be mentioned that the first laboratory prototype was fabricated for short distance ranges, about $165 \mathrm{~cm}$, and the circuit parameters were adjusted accordingly. For a larger distance, some of these parameters will need to be changed appropriately.

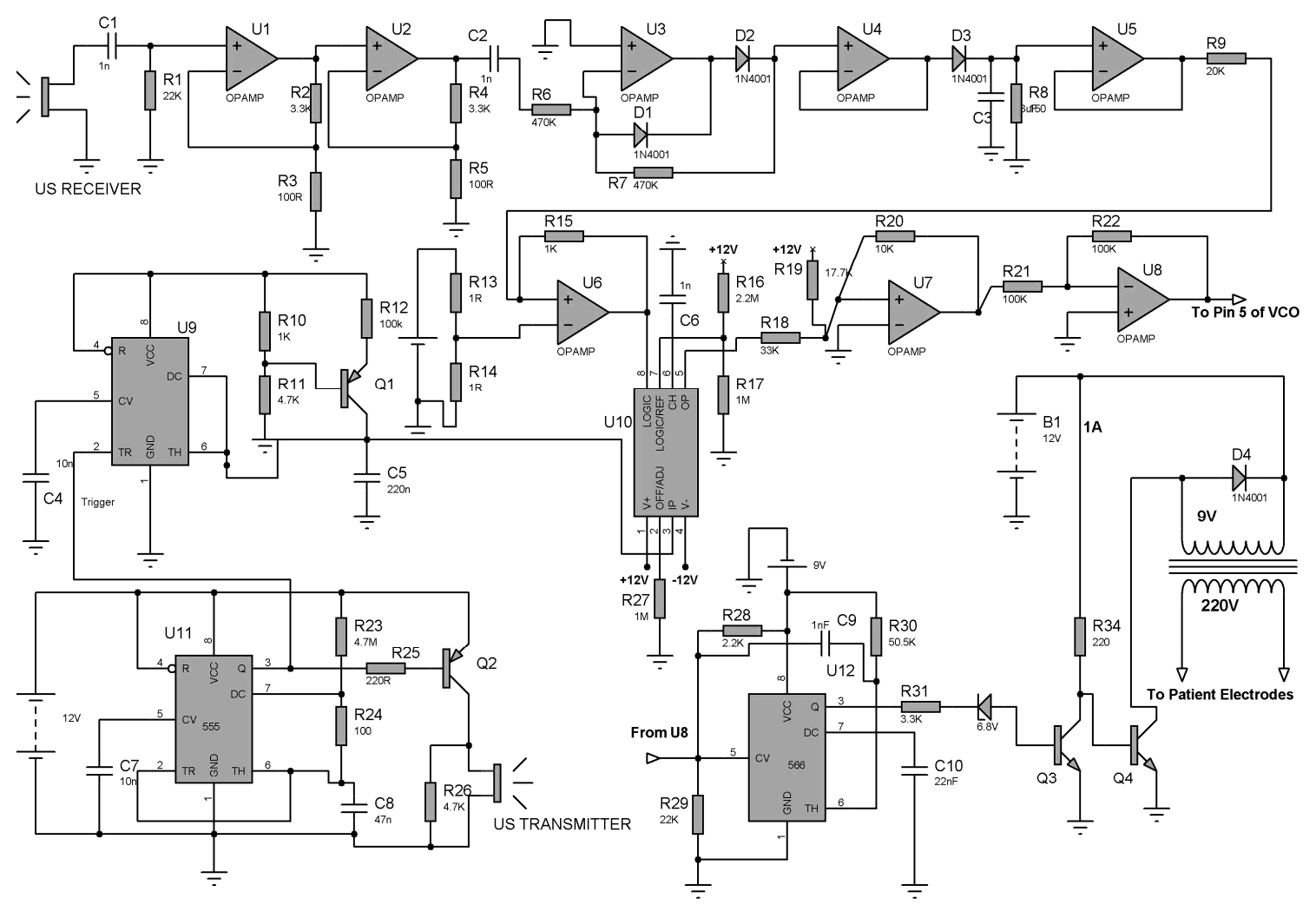

Fig 5: Circuit Diagram of the fabricated prototype

2.6.1 Pulsed Ultrasound Transmitter: This section is built around U11, a popular timer IC (555) to deliver free running pulses to a $40 \mathrm{kHz}$ ultrasound transducer through a driver transistor Q2. The pulse width should be considerably shorter than the natural time period of oscillation $(25 \mu$ s for $40 \mathrm{kHz})$ of the transducer to deliver a single short burst of damped sinusoids. If the pulse width is larger, two consecutive damped sinusoids would be generated at both the rising edge and the falling edge of the pulse and will complicate the time delay measurement. The repetition period of the pulse was designed based on the maximum distance that is intended to be covered by this device, and the frequency of information update for the user. As mentioned before, about $6 \mathrm{~Hz}$ was chosen for the repetition and the values of the circuit parameters were chosen accordingly. 
2.6.2 Triggered Ramp Generator: The above pulse generator for the transmitter triggers a ramp generator built around U9 which again uses a similar timer IC (555). As mentioned above the first laboratory prototype was designed for a short distance range, and so the period of the ramp generator was chosen accordingly. For a longer distance range, this period has to be changed appropriately. For this laboratory version, the range was chosen in such a way that the output of the ramp generator would rise from 0 to $8 \mathrm{~V}$ within $10 \mathrm{~ms}$. This time would correspond to a maximum range of $165 \mathrm{~cm}$ (considering twice the distance covered by the ultrasound pulse).

2.6.3 Receiving Amplifier And Pulse Shaper: The transmitted ultrasound pulse is usually in the form of a damped sinusoid because of the natural frequency of oscillation of the transmitting transducer. On the other hand the inertial characteristic of the receiving transducer contributes to a gradual increase of the waveform at the beginning, as discussed before. Thus the resulting received electrical pulse is in the form of a $40 \mathrm{kHz}$ wave packet with a gradually rising and then a gradually decreasing amplitude pattern. The electronics at the receiving end has to create a square pulse from this $40 \mathrm{kHz}$ wave packet. The amplifiers are built around U1 andU2 which are straightforward non-inverting amplifiers with high pass filters on both sides to minimize unwanted mains borne $(50 \mathrm{~Hz})$ noise and dc offset voltages. Common opamps (TL074) that were used for the present design do not have much open loop gain at 40kHz, therefore two stages were used. The next stages, built around U3, U4 and U5, rectify and smooth the signal to produce a dc pulse output which is further refined using a Schmitt trigger, built around U6. The output provides a trigger for the sample and-hold $(\mathrm{S} / \mathrm{H})$ circuit.

2.6.4 S/H Circuit And Level Shifter: On getting a trigger from the above circuit which corresponds to the time of receiving an echo pulse, this S/H circuit (U10) takes a sample of the voltage of the ramp generator mentioned above and holds it at that value for a certain time (a few $\mathrm{ms}$ ) for processing by the next circuit. This output is level shifted using U7 and U8 to suit the requirement of the VCO that follows. If no input comes due to absence of any object in the vicinity (no echo) the S/H output resets to the base value automatically after about 1.5 seconds waiting for the next trigger.

2.6.5 Voltage Controlled Oscillator (VCO): There are two conditions that have to be fulfilled for the proper functioning of the VCO IC (SE566, marked U12). One of the conditions is that the modulating voltage, coming from the output of U8, should be between three-fourth supply voltage and the supply voltage. Hence it is necessary to take the sampled voltage coming from the S/H circuit to the necessary range given by the condition. This calls for the use of a level shifter prior to the VCO as mentioned above. The components linked to the VCO IC were chosen and adjusted so that echoes from practically nearest target objects would give a frequency of $500 \mathrm{~Hz}$ and that from the most distant ones (as designed), about $50 \mathrm{~Hz}$.

2.6.6 Nerve Stimulator: For stimulation of nerve using skin surface electrodes at the wrist, where the nerve runs just below the skin, voltage pulses between $100 \mathrm{~V}$ and $200 \mathrm{~V}$ are necessary to produce adequate stimulation. This high voltage has been obtained using an off-the-shelf step down transformer (220V to $9 \mathrm{~V})$ connected in the reverse step-up mode. Two transistors (Q3 and Q4) were used in sequence to provide adequate current amplification for driving the transformer. The output of the transformer gives the necessary stimulating pulses to the human body through metal electrodes.

\section{RESULTS \& DISCUSSIONS}

The performances of each section were measured separately to find out whether these give the desired outputs. When these individual performances were satisfactory, the circuit parts were combined for the final test of performance. A target object was placed at various distances from the ultrasound transducers, both of which were kept at the same level. However, care was taken so that direct output from the 
transmitter does not reach the receiver. The time delay between transmitted and received echo pulses, voltage output of the ramp generator, and the frequency output of the VCO, which is the effective nerve stimulating frequency, were measured. The variations of measured pulse delay time and stimulating frequency with distance are shown in figures 6 and 7 respectively.

It may be seen that the time delay $t_{d}$ of echo pulse varies linearly with distance in Fig. 6 which is expected. That the VCO section is also performing well can be seen from the linear graph, but with negative slope in Fig.7. The experimental prototype, built up on a circuit prototyping breadboard, was designed for short distances to test the effectiveness of the developed circuitry. Tests made up to a distance of about $20 \mathrm{~cm}$ show a good linearity between the nerve stimulation frequency and the distance of the target object, which shows a success of the method.

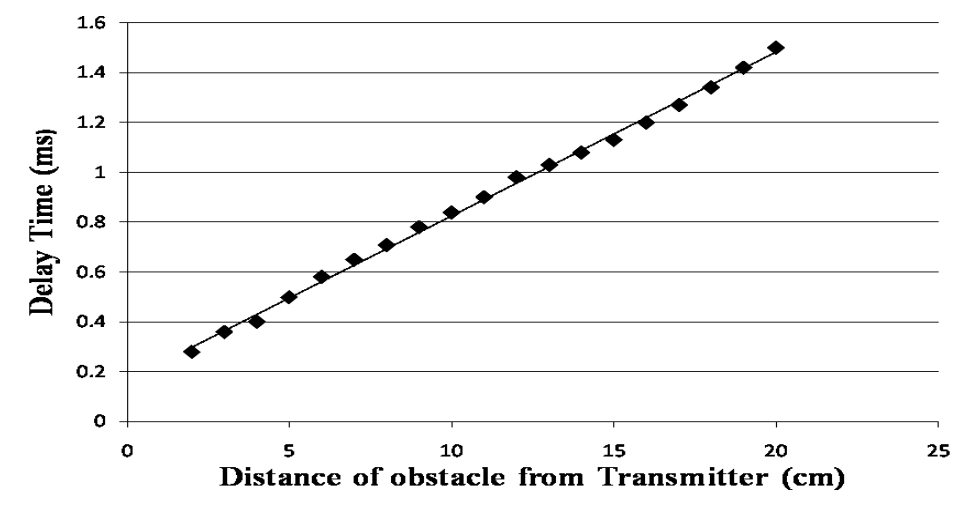

\section{CONCLUSIONS}

Technological aids such as the one developed in the present work for the blind may improve the quality of life of such physically disadvantaged people to a great extent. It is rather unfortunate that not much effort is given to develop such technologies, which is apparent from the very limited availability of these devices in the market. Again, whatever is available from the technologically advanced countries, are too

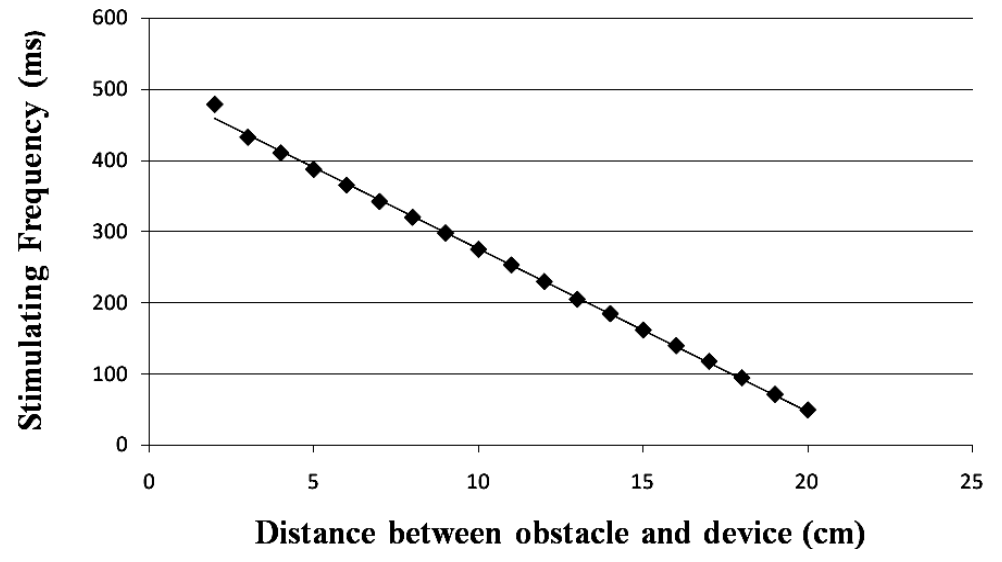

Fig 7: Measured Stimulating Frequency Against Distance expensive and unaffordable to the majority of potential users in the Third World. Besides, such devices from advanced countries fall short of the requirements of sustainability in a Third World country since the design is not often appropriate for the target region. Besides, repair and maintenance in a country remote from that of the manufacturer becomes a practical impossibility. Therefore, attempts to develop such technologies in the Third World itself are necessary viewed from the points of both cost and sustainability.

As already mentioned, going for nerve stimulation in presenting the deciphered information to a blind person makes a significant improvement over existing technologies that deliver the information through the ear. Therefore, this technology will also be useful for the advanced countries of the world.

This new technique does not curtail the natural faculty of hearing which a blind person has, a faculty which often is enhanced to discern subtle information that a normal person cannot. The ultrasound transducers may be mounted on a spectacle frame allowing the user to scan the surroundings by rotating 
and moving the head, thus being able to judge the size of objects in the surroundings, and the way they are moving, to some extent. The nerve stimulations may be applied at any point where the nerve fibres run just underneath the skin. As a nearest point of stimulation one may be inclined to use the facial nerve, however, it may be rather unpleasant to the user. Other options may be the radial nerve in the upper arm, median and ulnar nerves at the elbow, or the last two nerves at the wrist. All the points except the wrist have muscle around and fatty layers under the skin. The former may cause interruption of stimulation due to muscle moving in under the electrodes, while the latter will demand much higher currents for stimulation, making it rather unpleasant and painful. Therefore, wrist appears to be the best place for stimulation having no muscle and a minimum of fat layer, although this will require a long wiring from the spectacle frame to the wrist electrodes. Placing the whole device including the transducers and electronics on the wrist is another option, however, natural movement of the hand will disturb the echo pulsing process. Using wireless signal transfer between the transducer circuitry and the stimulator is another option, and appears to be the best, but will make the device more complex and expensive. The experimental prototype, built on a circuit prototyping breadboard, was designed for short distances to test the effectiveness of the developed circuitry. Tests made up to a distance of about $20 \mathrm{~cm}$ show a good linearity between the nerve stimulation frequency and the distance of the target object, which shows a success of the method. For more practical usage, the range of the device has to be increased to about $10 \mathrm{~m}$. At such distances, the received signal will be very weak and adequate care has to be exercised in reducing interference and noise as much as possible. This will need housing the transducers and the electronic circuitry in properly shielded enclosures, and having shielded cables for signal transmission between different segments of the device. In future more information may be incorporated, such as the nature of the obstacle, based on the amplitude of the received pulse, which will be made to alter the intensity of stimulation. For this a time varying gain in the receiving pulse amplifier (called a swept gain amplifier) would be needed as used in traditional ultrasound scanners.

Furthermore, for objects lying at the back of the user, an additional system may be placed with transducers facing the opposite way. Besides, it may be considered whether three dimensional positional information may be obtained using two or three transmitter-receiver pairs and computational techniques.

Thus the present work has taken a new step to develop a useful aid for the blind which will give the person more freedom in movement while avoiding obstacles and dangers.

\section{REFERENCES}

1. Tohru Ifukube, Tadayuki Sasaki, and Chen Peng, "A Blind Mobility Aid Modeled After Echolocation of Bats", IEEE Transactions on biomedical engineering, vol. 38, No. 5. pp. 461- 465, 1991

2. Bitjoka Laurent and Takougang Noupowou Alain Christian, "A sonar system modelled after spatial hearing and echolocating bats for blind mobility aid", International Journal of Physical Sciences, Vol. 2, pp. 104-111, 2007

3. Barshan B, Kuc R “A bat like sonar system for object localisation.”, IEEE Transactions on Systems, Man and Cybernetics. Vol 22, pp. 636-646, 1992

4. Heyes AD "The Nottingham obstacle detector: a technical description.", J. Vis. Impair Blind," Vol. 75, pp. 206209,1981

5. G S Dodgson, B H Brown, I L Freeston and J C Stevens, "Electrical stimulation at the wrist as an aid for the profoundly deaf", Clin. Phys. Physiol. Meas., vol. 4, pp. 403-416,1983.

6. M Muwyid Uzzaman Khan, M Shamiul Fahad and K Siddique-e Rabbani, "Ultrasound mobility aid for the blind using ultra frequency modulated nerve stimulation", $6^{\text {th }}$ International Conference on Electrical \& Computer Engineering, ICECE, Dhaka, Bangladesh. (Paper Id: p102; page: 171-174; IEEE Catalog Number: CFP 1068A-CDR; ISBN-978-4844-6279-7), 2010 